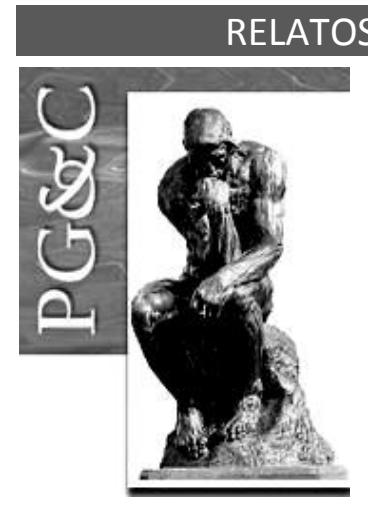

\title{
REDES INTERORGANIZACIONAIS E A CRIAÇÃO DE CONHECIMENTO: BUSCA SISTEMÁTICA
}

\author{
Karoline Brasil de Oliveira Ezequiel \\ Mestra em Desenvolvimento Socioeconômico pela Universidade do \\ Extremo Sul Catarinense, Brasil. \\ E-mail: karol brasill@hotmail.com \\ Cristina Keiko Yamaguchi \\ Doutora em Engenharia e Gestão do Conhecimento pela Universidade \\ Federal de Santa Catarina, Brasil. Professora da Universidade do Planalto \\ Catarinense, Brasil. \\ E-mail: criskyamaguchi@gmail.com
}

Melissa Watanabe

Doutora em Agronegócios pela Universidade Federal do Rio Grande do Sul, Brasil. Professora da Universidade do Extremo Sul Catarinense, Brasil.

E-mail: melissawatanabe@unesc.net

\begin{abstract}
Resumo
Estudos sobre redes interorganizacionais têm se desenvolvido com diferentes perspectivas, na busca de tentar compreender sua importância para a competitividade e inovação. Na economia do conhecimento, trabalhos sobre criação do conhecimento também possuem este foco. No entanto, pouco se sabe sobre como ocorre a criação do conhecimento em rede e qual a importância para as organizações. Desta forma este trabalhou objetivou conhecer o panorama das publicações sobre criação do conhecimento em redes interorganizacionais. Para isso, realiza busca sistemática na base de dados Scopus, escolhida por abranger uma gama expressiva de estudos sobre o tema. Assim, utiliza uma abordagem qualitativa, com objetivo de pesquisa exploratório. Diante das análises realizadas, dos artigos selecionados, compreende-se que as redes interorganizacionais precisam criar espaços e momentos, ba, que são espaços físicos, virtuais ou emocionais onde ocorrem a criação do conhecimento, que sejam favoráveis para a criação do conhecimento. Isto é importante, pois, percebese que a criação do conhecimento em rede contribui para a inovação das organizações participantes e aumentam suas vantagens competitivas.
\end{abstract}

Palavras-chave: Gestão do conhecimento. Criação do conhecimento. Redes interorganizacionais.

\section{INTERORGANIZATIONAL NETWORKS AND THE CREATION OF KNOWLEDGE: SYSTEMATIC SEARCH}

\begin{abstract}
Studies on interorganizational networks have developed from different perspectives, in the attempt to understand their value towards competitiveness and innovation. In today's knowledge economy, studies on knowledge creation also have this focus. However, little is known about how the creation of knowledge in network and how important to organizations. Therefore this work aimed to know the panorama of the publications on knowledge creation in interorganizational networks. In this regard, a systematic search was carried out in the Scopus database using a qualitative approach, as for the objective, it was designed as an exploratory study. Given the selected articles' analyzes, it was possible to understand that interorganizational networks need to create spaces and moments, $B a$, that are favorable for the creation of knowledge. An important aspect, since, it was noticeable that the creation
\end{abstract}

Perspectivas em Gestão \& Conhecimento, João Pessoa, v. 9, n. 1, p. 122-137, jan./abr. 2019. DOI: http://dx.doi.org/10.21714/2236-417X2019v9n1p122

http://periodicos.ufpb.br/ojs2/index.php/pgc. ISSN: 2236-417X. Publicação sob Licença (cc) EY-NC-ND 
of networked knowledge contributes to the innovation of the participating organizations and increases their competitive advantages.

Keywords: Knowledge management. Knowledge creation. Interorganizational networks.

\section{INTRODUÇÃO}

Estudos sobre redes interorganizacionais têm se desenvolvido com diferentes perspectivas, na busca de tentar compreender sua importância para a competitividade (VERSCHOORE; BALESTRIN, 2008; BRANDÃO et al., 2015) inovação (TÁLAMO; CARVALHO, 2010), bem como, descrever suas características e tipologias (OLIVEIRA; GUERRINI, 2002). Na economia do conhecimento, trabalhos com foco organizacionais se apoiam também na teoria da gestão do conhecimento, para a inovação e para a competitividade (GONÇALVES; VASCONCELOS, 2011; SOUZA, 2006).

Neste trabalho entende-se que as redes são importantes por meio da lógica dos laços fracos e fortes de Granovetter (1973), a qual compreende que indivíduos precisam interagir com pessoas fora do seu convívio diário para adquirir novos conhecimentos. Essa aquisição de conhecimento será analisada com base na teoria de Nonaka e Takeuchi (1997) sobre criação do conhecimento que ocorre, de acordo com os autores, como em uma espiral com a interação do conhecimento tácito, o qual está intrínseco no indivíduo, e o conhecimento explícito que pode ser facilmente adquirido, pois já foi transformado em algum documento, áudio, vídeo e afins.

Fica claro na literatura de Ahmadijan (2008) que os autores Nonaka e Takeuchi ao falarem sobre criação do conhecimento, apenas pincelaram sobre como este processo ocorre em nível interorganizacional. Sendo assim, alguns estudos sobre redes e criação do conhecimento (BALESTRIN; VERSCHOORE, 2016; AHMADIJAN, 2008) começaram a ser explorados a partir dai, com o intuito de entender a importância da criação do conhecimento entre organizações, principalmente as organizações de micro e pequeno porte, para o seu desenvolvimento.

Assim este trabalho buscou, conhecer o panorama das publicações sobre criação do conhecimento em redes interorganizacionais a partir de uma busca sistemática com os dois temas. Com isto, este trabalho foi subdividido em introdução, fundamentação teórica com foco para redes interorganizacionais, que trata da forma como as organizações se relacionam e a criação do conhecimento, a qual busca entender os processos utilizados para a inovação e não apenas o armazenamento de conhecimentos já existentes. Por fim, segue-se com os procedimentos metodológicos a fim de ressaltar de que forma o trabalho foi realizado e que estrutura metodológica foi utilizada com intuito de garantir a veracidade dos dados alcançados, resultados e análise levantados e discutidos pelas pesquisadoras e a conclusão a qual responde o objetivo proposto, bem como, indaga sobre pontos importantes para futuras pesquisas.

\section{FUNDAMENTAÇÃO TEÓRICA}

Foram abordados temas como a teoria das redes, sua importância e como tem transformado as estruturas sociais e econômicas. Após esta abordagem dirige-se o foco a criação do conhecimento finalizando com a criação do conhecimento interorganizacional. Esta fundamentação apresenta autores clássicos sobre cada temática e como estas estão sendo estudadas separadamente. Após compreender os campos de estudos isolados, segue a busca

Perspectivas em Gestão \& Conhecimento, João Pessoa, v. 9, n. 1, p. 122-137, jan./abr. 2019. 
sistemática para conhecer os trabalhos que já relacionam as teorias e quais resultados alcançados.

\subsection{Redes interorganizacionais}

Os seres humanos logo quando são inseridos na sociedade, passam a participar de redes em seus cotidianos e criam relações de interesse as quais se alteram ou expandem ao longo da vida (TOMAÉL; ALCARÁ; CHIARA, 2005). A internet, redes sociais por convivência ou por outras formas de intermediação entre pessoas, organizações, redes empresariais, redes de distribuição, são exemplos de redes existentes na sociedade (NEWMAN, 2003).

As redes podem ser compreendidas como uma interconexão de nós, que são os indivíduos, organizações ou países envolvidos. Ao passo que os relacionamentos se expandem os nós vão se interligando e formando neste contexto um formato de rede (SOUSA, 2009). Para que as redes se formem se faz necessário que aconteça uma interação entre os indivíduos e que estes compartilhem suas informações e conhecimentos (MÜLLER; STRAUHS, 2015).

Esta relação tem sido importante para o meio empresarial. Sabe-se que, com a globalização, empresas fortes conseguiram transpor barreiras e aumentar ainda mais seus lucros, reduzindo os impostos, os custos de sua produção e comercializando mundialmente. Porém, apesar da globalização trazer inúmeras vantagens, pequenas e micro empresas enfrentam dificuldades em competir com essas empresas (BECK, 1990). Assim podemos perceber a adoção de estratégias, por parte destas empresas, para se fortalecerem no mercado, como exemplo, se inserirem em redes interorganizacionais.

Estas redes, segundo Tavares (2008) são formadas por relacionamentos de cooperação empresarial. Por meio destas, empresas conseguem se unir e alcançarem benefícios em conjunto, o que dificilmente conseguiriam individualmente, e assim, se tornarem mais competitivas no mercado. Muitos empresários já conseguem perceber a importância desta relação e passam enxergar até concorrentes como aliados em alguns objetivos comuns e assim ficarem a frente de grandes empresas.

Estas redes têm como função primordial contribuir para o desenvolvimento de cada empresa, sem que precisem estar interligadas financeiramente (OLIVEIRA; FARIAS FILHO, 2005). A interação das empresas, pode estar relacionada a união de esforços para conseguirem diminuir os custos com capacitações e palestras, minimizar as ameaças do mercado, e também desenvolverem pesquisas cooperativas (WEGNER; DAHMER, 2004). É por meio deste processo que muitas empresas têm buscado desenvolver inovações (MÜLLER; STRAUHS, 2015).

Porém, a cooperação entre as empresas, nem sempre terá um bom resultado (SILVA; et al., 2015). Existem alguns motivos que enfraquecem a formação das redes e fazem com que as empresas não se adaptem com esse tipo interação. Uma pesquisa realizada com 140 empresas participantes de redes no Rio Grande do Sul, Brasil, percebeu que a confiabilidade é um dos fatores que mais importam para o fortalecimento de redes, pois muitos empresários possuem dificuldade de confiar nos demais e isso impactou no relacionamento destes (KLEIN; PEREIRA, 2014).

No entanto, Granovetter (1973), sociólogo americano expoente da abordagem da Nova Sociologia Econômica, apresenta vantagens da participação em rede por meio do conceito de laços fortes e laços fracos, sendo o primeiro composto por relacionamentos com pessoas próximas, como familiares e amigos e o segundo, apenas conhecidos, como ex-colegas de faculdade. Com esta teoria, o autor ressalta a importância dos laços fracos para adquirir novas informações, devido a sua convicção de que pessoas que estão em um mesmo contexto social (laços fortes) possuem os mesmos conhecimentos e informações.

Perspectivas em Gestão \& Conhecimento, João Pessoa, v. 9, n. 1, p. 122-137, jan./abr. 2019. 
Desta forma, aqueles que possuem poucos laços fracos estarão menos propensos a inovar, em virtude de estarem confinados apenas a informações e visões de seus próximos, o que pode tornar indivíduos ou organizações isolados das últimas novidades e os colocarem em posição de desvantagem no mercado de trabalho. $O$ fato de não possuir contatos além dos laços fortes pode prejudicar também na participação ou organização de movimentos políticos de qualquer tipo e que podem ser cruciais para os negócios (GRANOVETTER, 1983). O que mostra que transpor a barreira da falta de confiança pode contribuir para o desenvolvimento das organizações e indivíduos, e também facilita a criação de conhecimento explicitada na seção a seguir.

\subsection{Criação do conhecimento}

O significado de conhecimento tem gerado discussões ao longo do tempo, Nonaka e Takeuchi (1997) afirmam que durante o período grego já havia discussões filosóficas e epistemológicas. Entretanto, foi no final do século XX que o tema ganhou destaque sendo evidenciado por teóricos socioeconômicos de referência como Peter Drucker e Alvin Toffler, bem como, autores com foco organizacional os quais ressaltavam a importância deste recurso na nova economia que surgia (NONAKA; TAKEUCHI, 1997). Assim, evidenciava-se a passagem da era industrial para uma nova era do conhecimento, em que o conhecimento passa a ser reconhecido como o bem mais valioso para as organizações (DRUCKER, 1999).

Neste novo tempo, a gestão do conhecimento é vista como a base para as organizações que querem evoluir e inovar. Contudo, para que sejam bem sucedidas, é preciso entender que seu resultado ocorre pela junção de diversos fluxos de conhecimentos especializados (CHOO, 2000). Para Yamaguchi (2013) a gestão do conhecimento ocorre por meio da criação e compartilhamento do conhecimento, que lhe garanta a eficiência no mercado de forma sustentável.

O conhecimento pode ser tácito ou explícito, o primeiro é adquirido por meio de experiências, está na mente dos indivíduos e não é fácil de identifica-lo. Já o conhecimento explícito está formalizado em documento, fotos, vídeos, entre outros, sendo mais fácil seu acesso. Dentro do processo de criação os dois interagem e assim passam do nível individual, para o grupal, organizacional até o interorganizacional em um formato de espiral (NONAKA; TAKEUCHI, 1997).

Essa interação do tácito para explicito nos diferentes níveis ontológicos, citados acima, dentro da espiral ocorre por quatro formas, sendo a primeira socialização, em que o conhecimento tácito é compartilhado, de tácito para tácito. A segunda forma é a externalização em que ocorre a transformação do conhecimento tácito em explícito. Seguindo, tem-se a combinação, na qual o novo conhecimento explícito é combinado com o que já havia antes também explicitado. Por último, a internalização, momento que o novo conhecimento já explicito é difundido para os indivíduos e se transforma em conhecimento tácito (NONAKA; TAKEUCHI, 1997). Assim como está representado na Figura 1: 
Figura 1 - Espiral da Criação do Conhecimento Organizacional

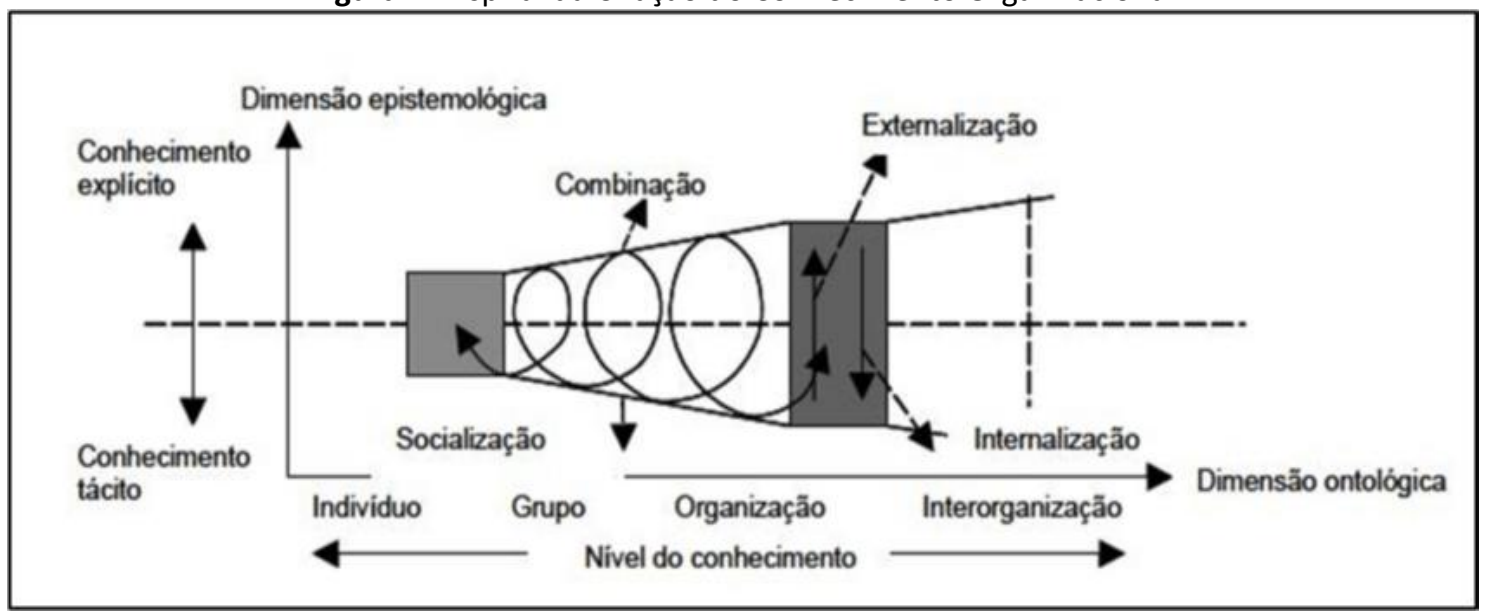

Fonte: Nonaka e Takeuchi (1997)

Este processo resulta na criação de novos conhecimentos, porém para que ocorra a conversão de conhecimento tácito e explicito é necessário um contexto físico. Este dentro do processo de criação do conhecimento pode ser considerado o ba. $O$ ba é o tempo e espaço fenomenológico em que ocorrem o compartilhamento do conhecimento (NONAKA; TOYAMA, 2008). É o espaço onde os indivíduos se relacionam e pode ser físico, virtual, mental ou uma combinação deles ao mesmo tempo e que resulta na criação do conhecimento (NONAKA; KONNO 1998).

Existe um modelo de ba para cada etapa do processo $\mathrm{SECl}$. O primeiro, que representa dentro do processo a fase da socialização, é o originating ba, local em que os indivíduos trocam experiências, compartilham sentimentos e emoções, apresentam seus modelos mentais. Nesta etapa cada um repassa o que sabe, sem julgamentos, apenas dividem-se conhecimentos tácitos. O próximo é o interacting ba, o qual relaciona-se com a etapa da externalização. Onde se encontram pessoas que possuem determinado conhecimento para algum projeto ou tarefa e discutem de forma mais aprofundada e assim se criam novos conceitos, regras e práticas (NONAKA; KONNO 1998). Segue-se então com o Cyber ba, espaço virtual que corresponde a etapa de combinação do modelo $\mathrm{SECl}$. Acredita-se que a combinação de novos conhecimentos explícitos com os já existentes se torna mais eficaz dispondo da tecnologia da informação. 0 último, é o exercising ba e está ligado a internalização. É o momento que o novo conhecimento explicito é transmitido para a organização sendo convertido em conhecimento tácito (NONAKA; KONNO, 1998). Conforme mostra a Figura 2: 
Figura 2 - Tipos de $b a$

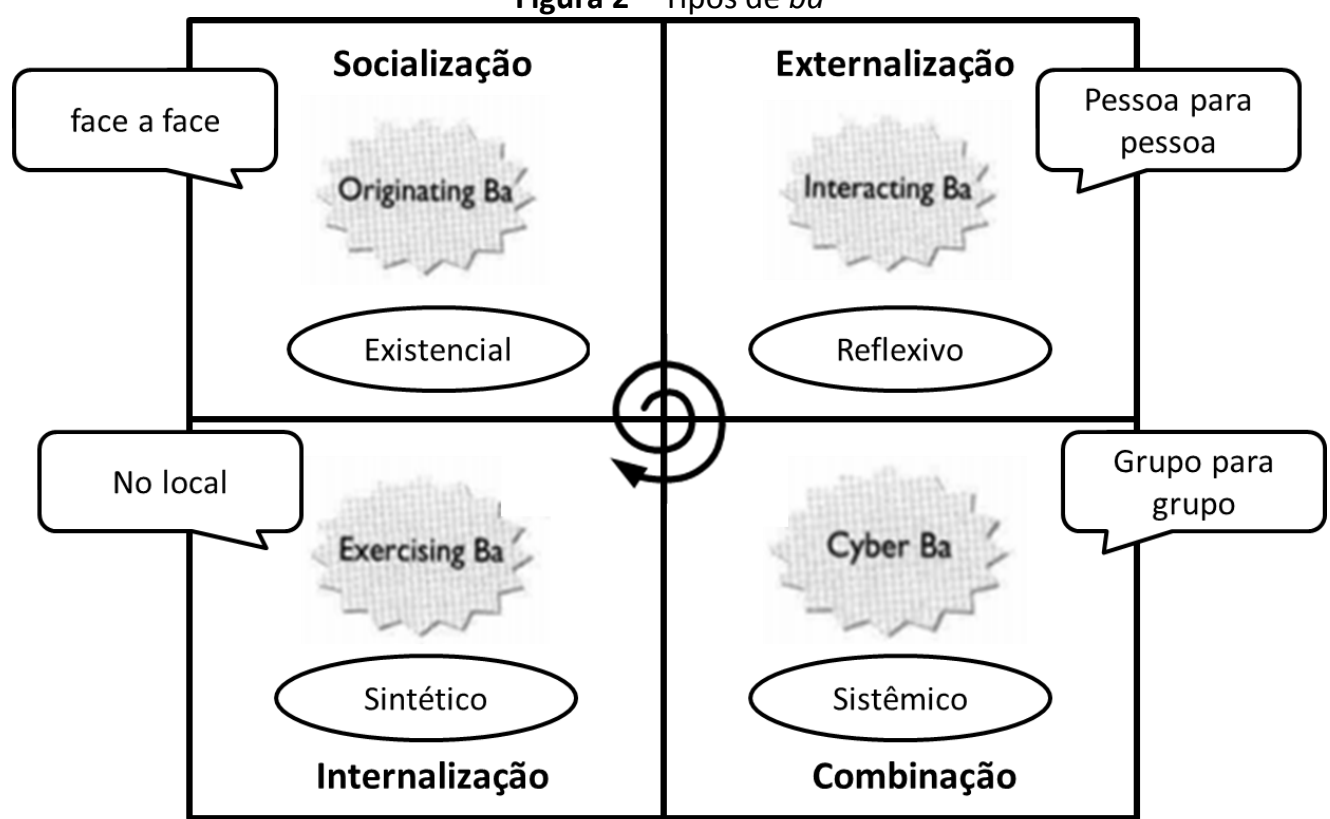

Onde:

Originating $\mathrm{Ba}=\mathrm{Ba}$ de origem;

Interacting $\mathrm{Ba}=\mathrm{Ba}$ de interação

Cyber $\mathrm{Ba}=\mathrm{Ba}$ Virtual

Exercising $\mathrm{Ba}=\mathrm{Ba}$ de exercício

Fonte: Nonaka e Konno (1998)

Nota: Tradução nossa

Dentro deste processo surgem quatro tipos de ativos de conhecimento, experiencial; conceitual, sistêmico e de rotina. O primeiro refere-se às habilidades e know-how dos indivíduos, emoções e sentimentos. O conceitual constitui em conhecimento explícito por meio de figuras, símbolos e outros meios tangíveis. O conhecimento sistêmico pode ser transferido com facilidade e está relacionado a documentos, especificações de produtos, licenças e patentes, propriedades intelectuais. Por fim o conhecimento de rotina está relacionado ações e práticas internas da organização, cultura organizacional, rotina do dia-adia do negócio ou história da organização (NONAKA; TOYAMA; KONNO, 2000).

Todas essas fases, além de acontecer dentro da organização, podem ocorrer também entre organizações, dentro de redes. No entanto na literatura foi pouco comentada, como mostra a seção seguinte.

\subsubsection{Criação do conhecimento interorganizacional}

As organizações do século 21 precisam gerenciar as forças contraditórias do mercado, sendo estas, competição e cooperação, integração e desintegração, e criatividade e eficiência para assim garantirem sua sobrevivência e desenvolverem-se. Para isso é necessário que as empresas busquem meios de criar novos conhecimentos de maneira rápida e eficaz (NONAKA, TOYAMA, 2002).

A teoria da criação do conhecimento interorganizacional, foi brevemente comentada pelos autores Nonaka e Takeuchi (1997). Os mesmos ressaltavam a importância pela busca do conhecimento fora dos limites da organização nos momentos de incertezas. Isto porque para

Perspectivas em Gestão \& Conhecimento, João Pessoa, v. 9, n. 1, p. 122-137, jan./abr. 2019. 
os autores a inovação contínua é resultado da ligação entre conhecimentos externos e internos. Este processo ocorre de forma que o conhecimento adquirido fora é disseminado e armazenado dentro da empresa e assim utilizado para criar novas tecnologias e produtos.

Dessa forma, a criação do conhecimento em interação com o ambiente externo pode ser um diferencial para a empresa agregar vantagem competitiva (BALESTRIN; VARGAS; FAYARD, 2005). Na busca para entender o seu funcionamento Ahmadjian (2008), analisa dois casos de criação de conhecimento interorganizacional de sucesso, como da Toyota e Vale do Silicio e alega que os mesmos fundamentos da criação do conhecimento organizacional são utilizados para o interorganizacional, bem como, se fortalece ainda a ideia da busca por um ba adequado. Da mesma forma Menezes et al (2017) corrobora quando afirma que a gestão do conhecimento se interconecta e contribuem para o desenvolvimento da empresa por meio da aprendizagem, da informação, do treinamento, do trabalho colaborativo e da utilização de técnicas criativas. Wons et al (2018) apontam que as maiores barreiras são individual: a falta de tempo, confiança, mentalidade individualista; e na barreira organizacional: falta de infraestrutura e recursos, ausência de cultura organizacional saudável, idioma, limites/distância física e conflitos de comunicação; e na barreira tecnológica: dificuldade no manuseio de ferramentas e sistemas e dificuldades em integrar e modificar os sistemas baseados em tecnologia. Dessa forma, é fundamental a conscientização sobre os benefícios do compartilhamento do conhecimento e de uso de técnicas que propiciem e facilitem a criação e a gestão do conhecimento nas empresas.

Para que a criação do conhecimento interorganizacional tenha sucesso, é necessário um ambiente propício para que os indivíduos compartilhem suas experiências, sentimentos, emoções que não se detenha apenas aos limites da empresa (BALESTRIN; VARGAS, FAYARD, 2005). Este tema, no entanto, ainda é pouco discutido na literatura. Uma breve busca sistemática nas bases de dados Spell, Scielo e Scopus, no dia 23 de março de 2017, com a palavra tanto no português quanto no inglês "inter-organizational knowledge creation", "interorganizational knowledge creation" e "creation of interorganizacional knowledge", identificou apenas 9 trabalhos, todos no repositório da Scopus.

Por isso, faz-se necessária ainda estudos para conhecer como a temática abordada vêm sendo trabalhada no Brasil e se as redes interorganizacionais brasileiras são eficazes no processo de criação do conhecimento.

Desta forma, neste trabalho, buscou compreender o panorama das publicações brasileiras sobre redes e criação do conhecimento.

\section{PROCEDIMENTOS METODOLÓGICOS}

Esta pesquisa apresenta uma abordagem qualitativa, com objetivo de pesquisa exploratória, tem como estratégia de pesquisa um estudo bibliográfico e a técnica de pesquisa análise de dados.

A busca foi realizada no dia 1 de maio de 2017 e atualizado no dia 1 de fevereiro de 2018, por meio de filtros, com as palavras-chaves network* and "knowledge creation" e em seguida redes e "criação do conhecimento".

A base de dados selecionada para este processo foi a Scopus, sendo esta escolhida por sua representatividade internacional. A plataforma Scopus foi a base de dados consultada, em virtude de o repositório ser considerado a maior base de dados em número de resumos e citações, sendo composto por 53.000 .000 de registros, 21.915 títulos e 5.000 publicações. É constituído de mais de 1.200 revistas e mais de 500 anais de conferência (ELSEVIER, 2019). E pelo fato da plataforma Scopus indexarem uma das principais revistas da área de gestão do conhecimento e ciência da informação nacional e internacional (LACERDA; ENSSLIN; ENSSLIN, 2012).

Perspectivas em Gestão \& Conhecimento, João Pessoa, v. 9, n. 1, p. 122-137, jan./abr. 2019. 
O objetivo de por aspas ("') nas palavras-chave "knowledge creation" e "criação do conhecimento" foi de buscar apenas o termo completo, sem trazer artigos que apresentassem essas palavras separadamente. Na palavra network foi acrescentado o sinal $\left({ }^{*}\right)$ para que também buscasse artigos com as palavras networks e networking. Após esta etapa foram utilizados os filtros para artigos brasileiros que abordassem estudos organizacionais, a fim de entender a criação do conhecimento em redes interorganizacionais. O Quadro 1 apresenta detalhadamente as etapas de todo processo da pesquisa.

Quadro 1 - Constituição da Busca sistemática

\begin{tabular}{|c|c|c|}
\hline $\mathbf{N}$ & $\begin{array}{l}\text { Etapas Segundo a } \\
\text { Cochrane Handbook }\end{array}$ & Pesquisa \\
\hline 1 & $\begin{array}{l}\text { Elaboração de uma } \\
\text { pergunta bem definida }\end{array}$ & $\begin{array}{l}\text { Qual o panorama das publicações sobre criação do conhecimento em } \\
\text { redes interorganizacionais. }\end{array}$ \\
\hline 2 & $\begin{array}{l}\text { Localização e seleção de } \\
\text { estudos }\end{array}$ & Levantamento na base Scopus. \\
\hline 3 & $\begin{array}{l}\text { Avaliação crítica dos } \\
\text { estudos }\end{array}$ & $\begin{array}{l}\text { A avaliação dos estudos foi desenvolvida pelo seguimento dos filtros } \\
\text { pré-estabelecidos. }\end{array}$ \\
\hline 4 & Recolha de dados & $\begin{array}{l}\text { Foram selecionadas palavras-chave, tanto em português como em } \\
\text { inglês. As palavras em inglês foram validadas por meio da pesquisa de } \\
\text { estudos já publicados. A aplicação dos termos nos repositórios seguiu } \\
\text { processo de filtragem. }\end{array}$ \\
\hline 5 & $\begin{array}{l}\text { Análise e apresentação } \\
\text { dos dados }\end{array}$ & $\begin{array}{l}\text { Foi desenvolvida por meio do uso de ferramentas com quadros, onde } \\
\text { inicialmente foi realizado um panorama geral das publicações e depois } \\
\text { foi realizado um aprofundamento do conteúdo. }\end{array}$ \\
\hline 6 & $\begin{array}{l}\text { Interpretação dos } \\
\text { resultados }\end{array}$ & $\begin{array}{l}\text { Apresentado nas seções a seguir. A interpretação possibilitará uma } \\
\text { fazer uma relação do que tem sido estudo sobre os temas criação do } \\
\text { conhecimento e redes interorganizacionais. No entanto a mesma } \\
\text { apresenta risco com relação a limitação da pesquisa, com o uso de } \\
\text { apenas uma base de dados e a possibilidade de haver palavras-chave } \\
\text { diferenciadas. }\end{array}$ \\
\hline 7 & $\begin{array}{l}\text { Aperfeiçoamento e } \\
\text { atualização }\end{array}$ & $\begin{array}{l}\text { A atualização dos dados ocorreu no período dos dias } 1 \text { de fevereiro de } \\
2018 .\end{array}$ \\
\hline
\end{tabular}

Fonte: Baseado em Pocinho (2008)

Com a pergunta delimitada e os passos para a realização da busca definidos buscou primeiramente conhecer o panorama dessas publicações. Após esta etapa, foi feita a análise dos resultados de acordo com os trabalhos que se relacionavam a pergunta de pesquisa.

\section{RESULTADOS E ANÁLISE}

O primeiro processo de filtragem realizado, com as palavras-chave network*, knowledge creation, nesta ordem e depois com os filtros artigos, Brasil e selecionadas as áreas de Business, Management and accounting; Social Sciences; Decision Sciences; Economics, Econometrics and Finance, resultou em 45 artigos. A Figura 3 mostra o número de publicações sobre o tema proposto no período entre 2015 e 2017 : 


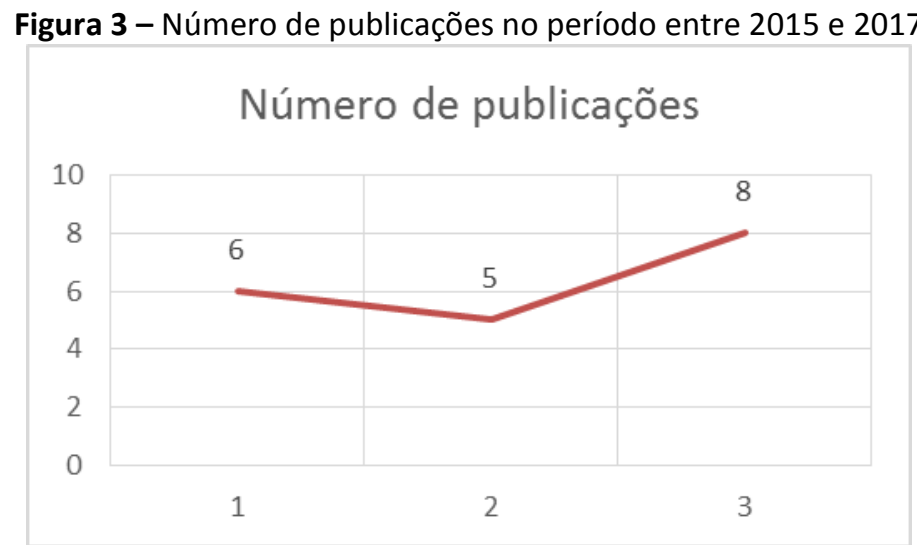

Fonte: Dados da pesquisa (2018)

A primeira publicação ocorreu em 2007 com 1 artigo publicado. Nos anos 2015, 2016 e 2017 ocorreram o maior número de publicações em revistas brasileira, com 6, 5 e 8 artigos publicados respectivamente.

Quadro 2 - revistas de maior publicação no período pesquisado

\begin{tabular}{|l|c|}
\hline Nome da revista & Número de artigos publicados \\
\hline Espacios & 9 \\
\hline Brazilian Administration Review - BAR & 3 \\
\hline Ciência da Informação & 3 \\
\hline Journal of Technology Management and Innovation & 3 \\
\hline
\end{tabular}

Fonte: Dados da pesquisa (2018)

A revista de maior publicação foi a Espacios com o resultado de 9 artigos, depois as revistas Brazilian Administration Review - BAR, Ciência da Informação e Journal of Technology Management and Innovation com 3 artigos cada, as demais apenas 2 ou 1 artigo.

Quadro 3 - universidades brasileiras que destacaram no tema da pesquisa

\begin{tabular}{|l|c|}
\hline Nome da universidade & Número de artigos \\
\hline Universidade de São Paulo & 7 \\
\hline Universidade federal de Santa Catarina & 5 \\
\hline Fundação Getúlio Vargas & 5 \\
\hline Universidade Federal do Rio Grande do Sul & 4 \\
\hline Universidade do Vale do Rio dos Sinos & 4 \\
\hline
\end{tabular}

Fonte: Dados da pesquisa (2018)

Referente a quantidade de artigos por instituição a Universidade de São Paulo se destacou com 7 artigos, a Universidade Federal de Santa Catarina e a Fundação Getúlio Vargas com 5 e a Universidade Federal do Rio Grande do Sul e a Universidade do Vale do Rio dos Sinos com 4 publicações.

As áreas escolhidas no processo de filtragem business, management and accounting representa $77,8 \%$ com 35 artigos. Seguido das Social Sciences com 16 artigos, 35,6\%. Este processo pode ser observado no Quadro 4: 
Quadro 4-Processo de Filtragem 1

\begin{tabular}{|l|l|c|c|}
\hline & \multicolumn{1}{|c|}{ Palavras-Chave } & $\begin{array}{c}\text { Scopus (Busca } \\
\text { 2017) }\end{array}$ & $\begin{array}{c}\text { Scopus (Busca } \\
\text { 2018) }\end{array}$ \\
\hline Filtro 1 & Network* (título, resumo ou palavras-chave) & 2.446 .247 & 2.655 .693 \\
\hline Filtro 2 & "Knowledge creation" (todos os campos) & 5.405 & 5.804 \\
\hline Filtro 3 & Artigos & 3.491 & 3.738 \\
\hline Filtro 4 & Brasil & 50 & 67 \\
\hline Filtro 5 & $\begin{array}{l}\text { Business, Management and accounting; Social } \\
\text { Sciences; Decision Sciences; Economics, } \\
\text { Econometrics and Finance. }\end{array}$ & 33 & 45 \\
\hline
\end{tabular}

Fonte: Dados da pesquisa (2018)

No segundo filtro foram encontradas publicações do ano de 2008 até 2017 . Sendo 2014 o ano com maior número, os quais correspondem a 3 artigos do total de 13 achados.

Quadro 5 - revistas de maior publicação no período pesquisado

\begin{tabular}{|l|c|}
\hline Nome da revista & Número de artigos publicados \\
\hline Journal Of Knowledge Management & 4 \\
\hline Journal Of Knowledge Management & 2 \\
\hline
\end{tabular}

Fonte: Dados da pesquisa (2018)

Quadro 6- universidades brasileiras que destacaram no tema da pesquisa

\begin{tabular}{|l|c|}
\hline Nome da universidade & Número de artigos \\
\hline Universidade de São Paulo & 2 \\
\hline University Center of United Metropolitan Faculties - FMU & 2 \\
\hline University of Toronto & 2 \\
\hline Fundação Getúlio Vargas & 2 \\
\hline Fundação Dom Cabral & 2 \\
\hline
\end{tabular}

Fonte: Dados da pesquisa (2018)

As áreas selecionadas e que tiveram maior número de artigos, foi a Business, management and accounting com 9 artigos e a Social Sciences com 7 artigos.

Quadro 7 - Processo de Filtragem

\begin{tabular}{|l|l|c|c|}
\hline & \multicolumn{1}{|c|}{ Palavras-Chaves } & $\begin{array}{c}\text { Scopus (Busca } \\
\text { 2017) }\end{array}$ & $\begin{array}{c}\text { Scopus (Busca } \\
\text { 2018) }\end{array}$ \\
\hline Filtro 1 & $\begin{array}{l}\text { "Knowledge creation" (título, resumo ou palavras- } \\
\text { chave) }\end{array}$ & 4.624 & 5.100 \\
\hline Filtro 2 & Network* & 2.285 & 2.513 \\
\hline Filtro 3 & Artigos & 1.336 & 1.456 \\
\hline Filtro 4 & Brasil & 15 & 20 \\
\hline Filtro 5 & $\begin{array}{l}\text { Business, Management and accounting; Social } \\
\text { Sciences; Decision Sciences, Multidisciplinary. }\end{array}$ & 12 & 13 \\
\hline
\end{tabular}

Fonte: Dados da pesquisa (2018)

Após a filtragem das publicações sobre redes e criação do conhecimento no Brasil, foi realizada a leitura dos 58 resumos de cada artigo encontrado. Nesta etapa alguns artigos foram identificados como trabalhos se repetiam e foram retirados e também que não correspondiam ao objetivo deste estudo por não relacionar os dois temas diretamente ou por não ser direcionado com o foco organizacional e por este motivo não aparecem na análise da pesquisa.

Perspectivas em Gestão \& Conhecimento, João Pessoa, v. 9, n. 1, p. 122-137, jan./abr. 2019. 
Sendo assim, restaram para análise apenas 8 artigos cujo perfil das publicações está apresentado no Quadro 3. Iniciam-se pelos artigos mais recentes, com a informação do repositório no qual se encontra o artigo, o autor, a instituição e o país da instituição, bem como o título deste e a revista em que foi publicado.

Quadro 8 - Perfil das publicações

\begin{tabular}{|c|c|c|c|c|c|c|}
\hline № & Ano & Autor & Instituição & País & Título & Revista \\
\hline \multirow[b]{2}{*}{1} & \multirow[b]{2}{*}{2017} & $\begin{array}{l}\text { Jordão, } \\
\text { R.V.D. }\end{array}$ & $\begin{array}{l}\text { Fundação Cultural Dr } \\
\text { Pedro Leopoldo }\end{array}$ & Brasil & \multirow{2}{*}{$\begin{array}{c}\text { Knowledge } \\
\text { management and } \\
\text { intellectual capital } \\
\text { in networks of } \\
\text { small- and medium- } \\
\text { sized enterprises }\end{array}$} & \multirow{2}{*}{$\begin{array}{l}\text { Journal of } \\
\text { Intellectual } \\
\text { Capital }\end{array}$} \\
\hline & & $\begin{array}{l}\text { Novas, } \\
\text { J.C. }\end{array}$ & University of Évora & Portugal & & \\
\hline \multirow[b]{2}{*}{2} & \multirow[b]{2}{*}{2015} & & $\begin{array}{l}\text { Fundação Pedro } \\
\text { Leopoldo }\end{array}$ & Brasil & \multirow{2}{*}{$\begin{array}{l}\text { Práticas de gestão } \\
\text { da informação e do } \\
\text { conhecimento em } \\
\text { pequenas e medias } \\
\text { empresas } \\
\text { organizadas em } \\
\text { rede: Um estudo } \\
\text { multicasos na } \\
\text { indústria Brasileira }\end{array}$} & \multirow[b]{2}{*}{$\begin{array}{l}\text { Perspectivas } \\
\text { em Ciência da } \\
\text { Informação }\end{array}$} \\
\hline & & $\begin{array}{l}\text { Jordão, } \\
\text { R.V.D. }\end{array}$ & $\begin{array}{l}\text { Center for Advanced } \\
\text { Studies in } \\
\text { Management and } \\
\text { Economics }\end{array}$ & Portugal & & \\
\hline \multirow[b]{2}{*}{3} & \multirow[b]{2}{*}{2015} & $\begin{array}{l}\text { Jenoveva } \\
\text {-Neto, R. }\end{array}$ & $\begin{array}{l}\text { Universidade Federal } \\
\text { de Santa Catarina - } \\
\text { UFSC }\end{array}$ & Brasil & \multirow{2}{*}{$\begin{array}{l}\text { Fatores internos e } \\
\text { externos que } \\
\text { influenciam a } \\
\text { capacidade } \\
\text { absortiva de } \\
\text { conhecimentos } \\
\text { voltados à inovação }\end{array}$} & \multirow[b]{2}{*}{ Espacios } \\
\hline & & $\begin{array}{l}\text { de Sá } \\
\text { Freire, P. }\end{array}$ & $\begin{array}{l}\text { Universidade Federal } \\
\text { de Santa Catarina - } \\
\text { UFSC }\end{array}$ & Brasil & & \\
\hline \multirow[b]{2}{*}{4} & \multirow[b]{2}{*}{2015} & $\begin{array}{l}\text { Do Canto } \\
\text { Cavalheir } \\
\text { o, G.M. }\end{array}$ & $\begin{array}{l}\text { Universidade Federal } \\
\text { Fluminense (UFF) }\end{array}$ & Brasil & \multirow{2}{*}{$\begin{array}{l}\text { Technology transfer } \\
\text { from a knowing } \\
\text { organization } \\
\text { perspective: An } \\
\text { empirical study of } \\
\text { the implementation } \\
\text { of a European } \\
\text { patent management } \\
\text { system in Brazil }\end{array}$} & \multirow{2}{*}{$\begin{array}{l}\text { World Review } \\
\text { of Science, } \\
\text { Technology } \\
\text { and } \\
\text { Sustainable } \\
\text { Development }\end{array}$} \\
\hline & & $\begin{array}{l}\text { Joia, } \\
\text { L.A. }\end{array}$ & $\begin{array}{l}\text { Getúlio Vargas } \\
\text { Foundation }\end{array}$ & Brasil & & \\
\hline \multirow{3}{*}{5} & \multirow{3}{*}{2014} & $\begin{array}{l}\text { Cunha, } \\
\text { F.J.A.P. }\end{array}$ & $\begin{array}{l}\text { Universidade Federal } \\
\text { da Bahia-UFBA }\end{array}$ & Brasil & \multirow{3}{*}{$\begin{array}{l}\text { Técnicas de } \\
\text { gerenciamento de } \\
\text { informações em } \\
\text { uma rede de } \\
\text { hospitais }\end{array}$} & \multirow{3}{*}{$\begin{array}{l}\text { Perspectivas } \\
\text { em Ciência da } \\
\text { Informação }\end{array}$} \\
\hline & & $\begin{array}{l}\text { Ribeiro, } \\
\text { N.M. }\end{array}$ & $\begin{array}{l}\text { Universidade da } \\
\text { Integração } \\
\text { Internacional da } \\
\text { Lusofonia Afro- } \\
\text { Brasileira - UNILAB } \\
\end{array}$ & Brasil & & \\
\hline & & $\begin{array}{l}\text { De } \\
\text { Barros } \\
\text { Pereira, } \\
\text { H.B. }\end{array}$ & $\begin{array}{l}\text { Universidade do } \\
\text { Estado da Bahia - } \\
\text { UNEB }\end{array}$ & Brasil & & \\
\hline 6 & 2013 & $\begin{array}{l}\text { Figueired } \\
\text { O, P.N. }\end{array}$ & $\begin{array}{l}\text { Brazilian School of } \\
\text { Public and Business } \\
\text { Administration } \\
\text { (EBAPE), Getúlio } \\
\text { Vargas Foundation }\end{array}$ & Brasil & $\begin{array}{l}\text { Embedding with } \\
\text { multiple knowledge } \\
\text { sources to improve } \\
\text { innovation } \\
\text { performance: The }\end{array}$ & $\begin{array}{l}\text { Knowledge } \\
\text { Management } \\
\text { Research and } \\
\text { Practice }\end{array}$ \\
\hline
\end{tabular}

Perspectivas em Gestão \& Conhecimento, João Pessoa, v. 9, n. 1, p. 122-137, jan./abr. 2019. 


\begin{tabular}{|c|c|c|c|c|c|c|}
\hline № & Ano & Autor & Instituição & País & Título & Revista \\
\hline & & & (FGV) & & $\begin{array}{l}\text { learning experience } \\
\text { of Motorola in Brazil }\end{array}$ & \\
\hline \multirow{3}{*}{7} & \multirow{3}{*}{2013} & $\begin{array}{l}\text { Caldas, } \\
\text { P.T. }\end{array}$ & $\begin{array}{l}\text { Federal University of } \\
\text { Campina Grande }\end{array}$ & Brasil & \multirow{3}{*}{$\begin{array}{c}\text { Inter-organizational } \\
\text { knowledge } \\
\text { conversion and } \\
\text { innovative capacity } \\
\text { in cooperative } \\
\text { networks }\end{array}$} & \multirow{3}{*}{$\begin{array}{c}\text { Journal of } \\
\text { Technology } \\
\text { Management } \\
\text { and } \\
\text { Innovation }\end{array}$} \\
\hline & & $\begin{array}{l}\text { Cândido, } \\
\text { G.A. }\end{array}$ & $\begin{array}{l}\text { Federal University of } \\
\text { Campina Grande }\end{array}$ & Brasil & & \\
\hline & & $\begin{array}{l}\text { da } \\
\text { Silveira, } \\
\text { T. }\end{array}$ & $\begin{array}{l}\text { Universidade Federal } \\
\text { do Rio Grande do Sul }\end{array}$ & Brasil & & \\
\hline \multirow{3}{*}{8} & \multirow{3}{*}{2008} & $\begin{array}{l}\text { Balestrin, } \\
\text { A. }\end{array}$ & $\begin{array}{l}\text { Universidade do Vale } \\
\text { do Rio dos Sinos - } \\
\text { UNISINOS }\end{array}$ & Brasil & \multirow{3}{*}{$\begin{array}{c}\text { Knowledge creation } \\
\text { in small-firm } \\
\text { network }\end{array}$} & \multirow{3}{*}{$\begin{array}{c}\text { Journal of } \\
\text { Knowledge } \\
\text { Management }\end{array}$} \\
\hline & & $\begin{array}{l}\text { Vargas, } \\
\text { L.M. }\end{array}$ & $\begin{array}{l}\text { Federal University of } \\
\text { Rio Grande do Sul - } \\
\text { UFRGS }\end{array}$ & Brasil & & \\
\hline & & $\begin{array}{l}\text { Fayard, } \\
\text { P. }\end{array}$ & Université de Poitiers & França & & \\
\hline
\end{tabular}

Fonte: Dados da pesquisa (2018)

Dessa amostra, o artigo intitulado "Knowledge creation in small-firm network" buscou entender como a dinâmica da criação de conhecimento ocorre dentro de uma rede de pequenas empresas. Dentre os resultados enfatizam que o contexto de uma rede de cooperação pode promover um ambiente de aprendizagem coletiva, de acordo com a criação de diferentes tipos de $b a$ que proporcionam a criação do conhecimento (BALESTRIN; VARGAS; FAYARD, P., 2008). Já Caldas e Cândido (2013) ao analisarem a dinâmica de conversão de conhecimento interorganizacional em espaços $b a$ existentes em uma rede cooperativa, não obtiveram resultados positivos. A rede estudada não apresentou uma configuração que favorecesse a conversão do conhecimento influenciando negativamente no desempenho da mesma.

No artigo "Embedding with multiple knowledge sources to improve innovation performance: The learning experience of Motorola in Brazil" as redes e conhecimento foram estudadas com intuito de identificar como ambos contribuem para inovação. A pesquisa explora como uma subsidiária de uma empresa multinacional (MNE) em uma economia emergente se integra com múltiplas fontes de conhecimento, tanto na sua rede corporativa (interna) quanto no país anfitrião (externo), para criar a capacidade de realizar atividades inovadoras. A pesquisa conclui que a subsidiária quando faz esforços para assimilar e internalizar o conhecimento por meio de rede, conhecimento externo, ela acumula progressivamente níveis mais altos de capacidades de inovação, o que possibilita que a mesma deixe de ser apenas um imitador, mas se transforme em um inovador a nível global (FIGUEIREDO, 2013).

Também em relação a rede para a inovação, uma pesquisa em uma rede de hospitais teve como objetivo mapear conceitos de inovação, de difusão de inovações e de aprendizagem, aplicados aos processos de inovação, além de revelar as ferramentas e fontes de informações para aprendizagem e inovações gerenciais incrementais em hospitais. A pesquisa mostrou que a rede contribui para a qualificação profissional e as relações entre sujeitos, porém ainda são pouco evidenciadas as interações entre os hospitais participantes, que seriam importantes para a socialização de novos conhecimentos e posterior internalização na rotina de cada hospital, sendo que prevalecem as interações intraorganizacionais (CUNHA; RIBEIRO; DE BARROS PEREIRA, 2014). 
A interação interorganizacional pode expandir as capacidades não-tradicionais dentro do grupo, por meio do processo de criação do conhecimento, bem como a adquirir expertise de fora do grupo, como mostrou a pesquisa de Do Canto Cavalheiro e Joia (2015) ao explorar a dinâmica das atividades de informação e conhecimento em um projeto de cooperação técnica envolvendo os escritórios de patentes europeus e brasileiros. Jenoveva-Neto e Sa-Freire (2015) salientam também que a empresa com capacidades de networking e uso de fontes externas de conhecimento e informações produzem valor agregado e inovação.

Jordão (2015) conclui seu estudo sobre a influência da organização em rede sobre as práticas de gestão do conhecimento de pequenas e médias empresas industriais brasileiras e apresenta que a rede influencia nos processos de criação do conhecimento, por meio das interações que ocorrem entre as organizações e que esse envolvimento traz benefícios. Também ressalta a importância do ba para a criação e o desenvolvimento de competências de indivíduos e empresas. Assim como salientando por Nonaka e Konno (1998), o ba são espaços e momentos, podendo ser físico, virtual ou mental, que contribuem para a interação e criação de conhecimentos.

No último artigo analisado, ainda, a criação, aquisição e manutenção de conhecimento e de capital intelectual se mostram importantes para redes de pequenas e médias empresas, as quais são influenciadas pelo contexto organizacional, formação da rede, cultura e estímulos para o compartilhamento de conhecimento (JORDÃO; NOVAS, 2017). De acordo com Beck (1990) apesar de a globalização trazer inúmeras vantagens, pequenas e micro empresas, as mesmas enfrentam também dificuldades em competir com essas empresas (BECK, 1990). Por isto a importância das redes, pois com a união e o compartilhamento do conhecimento, as organizações podem alcançar mercados e se tornarem mais competitivas, o que não seria possível caso se limitassem aos conhecimentos já existentes da organização ou se investissem em outros negócios sozinhas (TAVARES et al, 2008).

Um dos pontos de maior relevância levantados pelos artigos que faziam relação entre o tema redes e criação do conhecimento foi a importância do $b a$, o qual foi discutido por Nonaka e Konno (1998). Um dos artigos apresentam o ba como fator fundamental para o desenvolvimento de competências, tanto dos indivíduos quanto das empresas. Em outro caso, podem-se observar, também, relatos de que as redes são capazes de contribuir para a aprendizagem coletiva, bem como para a criação do conhecimento, desde que sejam criados diversos tipos $b a$. Também ficou evidente em um dos estudos que a rede a qual foi analisada não possuía um ba favorável para criação do conhecimento, quando afirmou que a mesma não apresentava uma configuração adequada e que isso afetava no seu desenvolvimento.

Fica claro que as redes precisam se preocupar em realizar a gestão do conhecimento e promover espaços que contribuam a criação do conhecimento, para que as organizações possam potencializar seus resultados. Mesmo em redes informais, é preciso que sejam criados ambientes favoráveis para que essas parcerias consigam refletir positivamente para o negócio. Sendo que é por meio das redes que muitas empresas buscam desenvolver inovações (MÜLLER; STRAUHS, 2015). O que ficou evidente é que organizações com capacidades de networking agregam valor e produzem inovações.

Em mercados altamente competitivos as empresas buscam novas formas de ganhar vantagem e se fortalecerem. As redes e a criação do conhecimento dentro delas, de acordo com a literatura podem contribuir com organizações que sozinhas não conseguem garantir recursos necessários para sua sobrevivência. Entretanto é necessário que as organizações que busquem firmar laços com agentes externos, além de proporcionar espaços que contribuam para a criação do conhecimento, ao mesmo tempo trabalhem também a confiança para que assim conquistem se desenvolvam e consigam resultados positivos. 


\section{CONSIDERAÇÕES FINAIS}

O presente artigo propôs conhecer o panorama das publicações sobre criação do conhecimento em redes interorganizacionais. Ciente de que a teoria deixa lacunas quanto a esta temática, pois pouco aborda sobre a criação do conhecimento fora dos limites das organizações, sendo importante para a organização que pretende inovar e desenvolver novas tecnologias, práticas e processos.

Diante das análises realizadas foi possível compreender que as redes interorganizacionais precisam criar espaços e momentos, a que denominamos de $b a$, que sejam favoráveis para a criação do conhecimento. Muller e Strauhs (2015); corrobora quando afirma que as redes são necessárias para que ocorra a interação entre os indivíduos e o compartilhamento das informações e conhecimento, contribui para a inovação das organizações participantes e aumentam sua vantagem competitiva (OLIVEIRA; FARIAS FILHO, 2005; TAVARES, 2008). Nesta nova era econômica as organizações precisam compreender que a cooperação pode trazer benefícios, principalmente para micro e pequenas empresas que não conseguem concorrer com grandes organizações.

Compreende-se que na era do conhecimento não é apenas o capital econômico que fazem as organizações alcancem âmbitos maiores no mercado, mas sim, quem possui capital intelectual e consegue gerenciá-lo. No entanto, para que esta participação seja eficaz, se faz necessária a confiança, para que não ocorram falhas na interação do conhecimento ou que o compartilhamento de conhecimento seja prejudicado (MENEZES et al, 2017; WONS et al, 2018).

Sugere-se que próximos estudos, ampliar as bases de dados visando ampliar a compreensão e inclusão de ações para verificar as características dos ba que promovem e quais conhecimentos são criados. Da mesma forma, incluir estudo de caso, nos quais possam ser evidenciados os resultados dessas pesquisas.

\section{AGRADECIMENTOS}

À Coordenação de Aperfeiçoamento de Pessoal de Nível Superior (CAPES) e ao Grupo de Estudo em Conhecimento, Inovação e Desenvolvimento (GECID).

\section{REFERÊNCIAS}

AHMADJIAN, C. L. Criação do Conhecimento Interorganizacional: Conhecimento e Redes. In: TAKEUCHI, H.; NONAKA, I. (orgs.). Gestão do conhecimento. Porto Alegre: Bookman, 2008. p. 201-216.

BALESTRIN, A.; VARGAS, L. M.; FAYARD, P. Criação de Conhecimento nas Redes de Cooperação Interorganizacional. RAE-Revista de Administração de Empresas, v. 45, n. 3, p. 52-64. 2005.

BALESTRIN, A.; VARGAS, L. M.; FAYARD, P. Knowledge creation in small-firm network. Journal of Knowledge Management, v. 12, n.2, p. 94-106, 2008.

BALESTRIN, A.; VERSCHOORE, J. Redes de cooperação empresarial: estratégias de gestão na nova economia. Porto Alegre: Bookman, 2016.

BRANDÃO, R. A.; GOMES, S. C.; TANGERINO NETO, D.; CABRAL, E. R. Redes de cooperação como indutores de ganhos de competitividade. Revista Periódica da Faculdade de Belém, Belém, v.4, n.1, p. 34-48, 2015.

Perspectivas em Gestão \& Conhecimento, João Pessoa, v. 9, n. 1, p. 122-137, jan./abr. 2019. 
CALDAS, P. T.; CÂNDIDO, G. A. Inter-organizational knowledge conversion and innovative capacity in cooperative networks. Journal of Technology Management and Innovation, v. 8, p. 104-114, 2013.

CHOO, C. W. Working with knowledge: how information professionals help organisations manage what they know. Library Mangement, v. 21, n. 8, p. 395-403, 2000.

CUNHA, F. J. A. P.; RIBEIRO, N. M.; DE BARROS PEREIRA, H. B. Técnicas de gerenciamento de informações em uma rede de hospitais. Perspectivas em Ciência da Informação, v.19, n.1, p.22-36, 2014.

DO CANTO CAVALHEIRO, G. M.; JOIA, L. A. Technology transfer from a knowing organization perspective: An empirical study of the implementation of a European patent management system in Brazil. World Review of Science, Technology and Sustainable Development, v. 12, n. 2, pp. 152-172, 2015.

ELSEVIER. Scopus: an eye on global research. Disponível em: https://www.elsevier.com/solutions/scopus\#. Acesso em 20 jan. 2019.

FIGUEIREDO, P. N. Embedding with multiple knowledge sources to improve innovation performance: The learning experience of Motorola in Brazil. Knowledge Management Research and Practice, v. 11 n.4, p. 361-373, 2013.

GONÇALVES, S. F. R.; VASCONCELOS, M. C. R. L. Práticas e Ferramentas de Gestão do Conhecimento no Âmbito da Administração Tributária de Minas Gerais: Oportunidade para uma Política Institucional. In: ENCONTRO NACIONAL DA ASSOCIAÇÃO NACIONAL DE PÓSGRADUAÇÃO E PESQUISA EM ADMINISTRAÇÃO, 35, 2011, Rio de Janeiro. Anais eletrônicos... Rio de Janeiro, 2011, p. 1-16. Disponível em: http://www.anpad.org.br/admin/pdf/GPR1256.pdf . Acesso em: 13 mar. 2018.

GRANOVETTER, M. The strength of weak ties. American Journal of Sociology, v. 78, n. 6, p. 1360-1380, 1973.

JENOVEVA-NETO, R.; FREIRE, S. P. Fatores internos e externos que influenciam a capacidade absortiva de conhecimentos voltados à inovação. Espacios, v. 36, n. 19, p. 3, 2015.

JORDÃO, R. V. D. Práticas de gestão da informação e do conhecimento em pequenas e médias empresas organizadas em rede: um estudo multicasos na indústria brasileira. Perspectivas em Ciência da Informação, v. 20, n. 3, p. 178-199, 2015. Disponível em: http://portaldeperiodicos.eci.ufmg.br/index.php/pci/article/view/1737. Acesso em: 02 fev. 2018.

JORDÃO, R. V. D.; NOVAS, J. C. Knowledge management and intellectual capital in networks of small- and medium-sized enterprises. Journal of Intellectual Capital, v. 18, n.3, p. 667-692, 2017.

LACERDA, R. T.; ENSSLIN, L.; ENSSLIN, S. R. Uma análise bibliométrica da literatura sobre estratégia e avaliação do desempenho. Gestão da Produção, São Carlos, v. 19, n. 1, 59-57, 2012.

Perspectivas em Gestão \& Conhecimento, João Pessoa, v. 9, n. 1, p. 122-137, jan./abr. 2019. 
MENEZES, K. C.; JOHAN, J.; VALENTIM, P. P.; SCOTT, P. Gestão do conhecimento nas organizações: Uma aprendizagem em rede colaborativa. Perspectivas em Gestão \& Conhecimento, João Pessoa, v. 7, Número Especial, p. 145-159, mar. 2017.

MÜLLER, R.; STRAUHS, F. R. Contribuições teóricas sobre o estudo de redes de conhecimento interorganizacionais. In: SIMPÓSIO NACIONAL DE CIÊNCIA, TECNOLOGIA E SOCIEDADE, 6, 2015, Rio de Janeiro. Anais eletrônicos... Rio de Janeiro: ESOCITE / TECSOC, 2015, p.1-20. Disponível em:

http://www.rio2015.esocite.org/resources/anais/5/1440788630 ARQUIVO CONTRIBUICOEST EORICASSOBREOESTUDODEREDESDECONHECIMENTOINTERORGANIZACIONAIS.pdf. Acesso em: 27 jul. 2016.

NONAKA, I.; KONNO, N. The concepto of "Ba": building foundation for Knowledge Creation. California Management Reviw, v. 40. n. 3. p. 40-54. 1998.

NONAKA, I.; TAKEUCHI, H. Criação do conhecimento na empresa: Como as empresas japonesas geram a dinâmica da inovação. 20 ed. Rio de Janeiro: Elsevier, 1997.

NONAKA, I.; TOYAMA, R. A firm as a dialectical being: Towards a dynamic theory of a firm. Industrial and Corporate Change, v. 11, n. 5. p. 995-1009, 2002.

OLIVEIRA, R. F. de; GUERRINI, F. M. Características das tipologias de redes de cooperação entre empresas. In: Encontro Nacional de Engenharia de Produção, 22, 2002, Curitiba. Anais Eletrônicos... Curitiba, 2002, p. 1-7. Disponível em: http://www.abepro.org.br/biblioteca/enegep2002 tr76 0616.pdf. Acesso em 22 set. 2016.

SOUZA, D. B. L. Gestão do conhecimento nas organizações: desafios e oportunidades. Revista Estação Científica, n. 3, p. 1-14. 2006. Disponível em: <http://portal.estacio.br/media/4376/5gestao-conhecimento-organizacoes-desafios-oportunidades.pdf >. Acesso em: 22 set. 2016.

TALAMO, J. R.; CARVALHO, M. M. de. Redes de cooperação com foco em inovação: um estudo exploratório. Gestão da Produção, São Carlos, v. 17, n. 4, p. 747-760, 2010.

VERSCHOORE, J. R.; BALESTRIN, A. Ganhos competitivos das empresas em redes de cooperação. Revista de Administração - Eletrônica, São Paulo, v.1, n.1, 2008.

WONS, L.; SOUZA, R. O. C.; SILVA, H. F. N.; FERNANDES, F. R. Barreiras ao compartilhamento do conhecimento nas organizações. Perspectivas em Gestão \& Conhecimento, João Pessoa, v. 8, número especial, p. 86-101, out. 2018

YAMAGUCHI, C.K. Contabilidade ambiental nas organizações: instrumento de criação do conhecimento. Curitiba: Juruá, 2013.

Artigo recebido em 19/03/2018 e aceito para publicação em 13/02/2019 